Research Paper

\title{
SATB1 Knockdown Inhibits Proliferation and Invasion and Decreases Chemoradiation Resistance in Nasopharyngeal Carcinoma Cells by Reversing EMT and Suppressing MMP-9
}

\author{
Dongni Zhou ${ }^{1}$, Chunsheng Ye², Zhiyong Pan ${ }^{2}$ and Yanfei Deng $2,3 \bowtie$ \\ 1. Department of Pathology, Zhongshan Hospital, Xiamen University, Xiamen, Fujian, China. \\ 2. Department of Otolaryngology-Head and Neck Surgery, Zhongshan Hospital, Xiamen University, Xiamen, Fujian, China. \\ 3. Union School of Clinical Medicine, Fujian Medical University, Fuzhou, Fujian, China. \\ $\square$ Corresponding author: Yanfei Deng, M.D., Ph.D., Department of Otolaryngology-Head and Neck Surgery, Zhongshan Hospital, Xiamen University, 209 \\ Hubin South Road, Xiamen, Fujian 361004, China. E-mail: dyf@xmzsh.com; dyanfei@139.com.
}

(C) The author(s). This is an open access article distributed under the terms of the Creative Commons Attribution License (https://creativecommons.org/licenses/by/4.0/). See http://ivyspring.com/terms for full terms and conditions.

Received: 2020.06.22; Accepted: 2020.10.09; Published: 2021.01.01

\begin{abstract}
Background: Special AT-rich sequence binding protein 1 (SATBI) is a chromatin organizer and transcriptional regulator which regulate numerous cellular processes through effects on multiple gene expression. SATBI is associated with drug resistance in several cancers. Whether SATBI involves radiation resistance in nasopharyngeal carcinoma (NPC) and underlying mechanism of SATBI to participate in chemoradiotherapy resistance in NPC have not been elaborated.

Methods: Chemoradioresistant NPC cell lines 5-8F/DDP (cisplatin) and 5-8F/R (radiation) were developed from 5-8F cell line. The expressions of SATB1, MMP-9 and EMT markers (Vimentin and E-cadherin) in these cell lines were examined by reverse transcription-quantitative (RT-q) PCR and western blot (WB) analysis. Cell viabilities of 5-8F/DDP treated with various concentrations of DDP and 5-8F/R irradiated with various doses of $X$-ray at the indicated time were investigated by MTT test. SATB1 was silenced in 5-8F/DDP and 5-8F/R cells by short hairpin RNA, and then the expressions of SATB1, MMP-9, Vimentin and E-cadherin were evaluated by RT-qPCR and WB analysis; the abilities of cell proliferation and invasion were assessed using MTT and transwell assays, respectively. Drug and radiation resistance assays were performed after SATBI knockdown and cell viability was detected by MTT method.

Results: SATB1, MMP-9 and Vimentin were markedly upregulated in 5-8F/DDP and 5-8F/R cells compared with 5-8F cell, whereas E-cadherin was obviously downregulated. 5-8F/DDP and 5-8F/R cells displayed drug and radiation resistance to DDP or X-irradiation, respectively, while DDP or X-irradiation inhibited 5-8F cell viability in a time- and dose-dependent manner. Subsequently, knockdown of SATBI resulted in decreased MMP-9 and Vimentin expression and increased E-cadherin expression in 5-8F/DDP and 5-8F/R. Furthermore, silencing of SATB1 suppressed proliferative and invasive abilities of 5-8F/DDP and 5-8F/R cells. Additionally, SATB1 knockdown reduced drug resistance of 5-8F/DDP cell to DDP and decreased radiation resistance of $5-8 \mathrm{~F} / \mathrm{R}$ cell to $\mathrm{X}$-ray.

Conclusion: These results suggest that high expression of SATBI plays an important role in the malignant behavior of NPC and leads to X-radiation and drug resistance in NPC through promoting EMT process and enhancing MMP-9 expression. SATB1 may be a promising therapeutic target for aggressive and chemoradiation resistant NPC.
\end{abstract}

Key words: Nasopharyngeal carcinoma, SATB1, MMP-9, Vimentin, E-cadherin, EMT, Chemoradiation resistance

\section{Introduction}

Nasopharyngeal carcinoma (NPC) is an southern China, southeast Asia, north Africa and epithelial malignancy with high prevalence in some Arctic regions and shows strongly aggressive 
and metastatic biological properties, leading to a relapse or poor prognosis [1, 2]. Until now, radiotherapy combined with chemotherapy remains as the preferred treatment for NPC $[2,3]$. Although great improvement has been achieved in therapeutic technology, residual or recurrent tumors remain as the outstanding cause of NPC treatment failure [2-4], especially for advanced stage patients. Alternatively, chemoradiation resistance is often induced and is a major factor leading to the failure of chemoradiotherapy and bad prognosis in NPC patients $[2,5,6]$. Therefore, even some patients with the same clinical stages have different treatment outcomes. With the development of gene-based precision medicines, it is an urgent need to explore potential therapeutic targets for NPC and identify valuable biomarkers to predict the chemoradiotherapy resistance of NPC. Nevertheless, the study in this aspect remains limited.

The special AT-rich sequence binding protein 1 (SATB1), a transcription factor and a global genome organizer, is involved in pathogenesis and progression of numerous human cancers [7, 8]. Aberrant expression of SATB1 has been found to be associated with proliferation, invasion, metastasis and poor prognosis in various cancers [8, 9]. SATB1 was also identified as an independent prognostic marker in many types of cancer $[8,10]$. Furthermore, previous study found that SATB1 contributed to multidrug resistance (MDR) phenotype in breast cancer cells [11]. Another study has confirmed that SATB1 contributes to MDR by inhibiting accumulation of vincristine (VCR) in gastric cancer cells and protecting the cells from VCR-induced apoptosis [12]. Subsequently, our recent studies have revealed that SATB1 is obviously upregulated in primary NPC tissues and NPC cell line 5-8F and linked to drug resistance in NPC cell line CNE-2 [13, 14]. However, to our knowledge, no data are available on the relationship between SATB1 and radiation resistance in NPC. Additionally, the underlying mechanism of SATB1 to participate in chemoradiotherapy resistance in NPC is yet to be elucidated.

The epithelial-to-mesenchymal transition (EMT) is characterized as a transition from the epithelial cell phenotype into a mesenchymal phenotype. EMT is a crucial event leading to the initiation of invasion and metastasis for tumor progression and involved in chemoradioresistance of tumor cells, including NPC $[15,16]$. The characteristics of EMT are the loss of epithelial surface markers, most notably E-cadherin, and the acquisition of mesenchymal markers including Vimentin. E-cadherin and Vimentin were frequently dysregulated in multiple human cancers $[16$, 17]. Recent findings have indicated that dysregulated SATB1 may mediate the reversion of EMT and mesenchymal-to-epithelial transition (MET) process and regulate its downstream target genes such as matrix metalloproteinases (MMPs; e.g. MMP-2, MMP-9) and EMT-related genes (e.g. E-cadherin, Vimentin) [18-20].

MMP-9, the important MMP family member, is a key protease to remodel and degradate extracellular matrix. It has been identified that MMP-9 plays a vital role in tumor development and progression [21]. Further investigations have also implicated that MMP-9 is closely associated with chemoradiation resistance in certain tumors $[22,23]$ and participates in EMT process [24]. For example, Asuthkar and his colleagues found that inhibition of MMP-9 resulted in suppression of EMT in medulloblastoma cells [25]. However, to the best of our knowledge, no reports are available on the relationship between MMP-9 and chemoradiation resistance of NPC.

In this study, we developed radioresistant and chemoresistant NPC cell lines to detect the expression of SATB1, MMP-9, Vimentin and E-cadherin; and investigate the effect of SATB1 knockdown by short hairpin RNA (shRNA) on NPC cell proliferation, invasion, chemoradiation resistance; and identify the influence of SATB1 silencing on expression of MMP-9, E-cadherin and Vimentin; and then explore the possible mechanisms of above situations.

\section{Materials and Methods}

\section{Cell lines and cell culture}

The human NPC cell line 5-8F was cultured in RPMI-1640 medium (Gibco, Grand Island, NY, USA) supplemented with $10 \%$ fetal bovine serum (Invitrogen, Carlsbad, CA, USA), $100 \mathrm{IU} / \mathrm{ml}$ penicillin (Sigma-Aldrich Corp., St. Louis, MO, USA) and 100 $\mu \mathrm{g} / \mathrm{ml}$ streptomycin (Sigma-Aldrich Corp.) at $37^{\circ} \mathrm{C}$ in a humidified atmosphere with $5 \% \mathrm{CO}_{2}$. Exponentially growing cells were used for all experiments. $5-8 \mathrm{~F}$ cell line used in this study was purchased from Cancer Institute, Central South University (Changsha, China).

\section{Establishment of a radioresistant cell line}

The generated procedures of radioresistant subline of $5-8 \mathrm{~F}$ were described and consulted in previous documents [26, 27]. In brief, the cells were first grown to approximately $70 \%$ confluence in $25-\mathrm{cm}^{2}$ culture flasks. Cells were initially irradiated with 2 Gy of $X$-ray from a linear accelerator (6 MV X-ray, UNIQUETM, Varian, Palo Alto, CA, USA) with a 1-cm tissue-equivalent bolus on top of the plate at a rate of $4 \mathrm{~Gy} / \mathrm{min}$ and then returned to the incubator. These cells did not receive another exposure dose until $70 \%$ confluence was reached. The fractional dose was gradually increased each time until 10 Gy was 
reached. The total dose was $60 \mathrm{~Gy}$. For the X-radiation treatment, cells were exposed to a desired dose, and were harvested in the indicated time points. The subline cells were named as $5-8 \mathrm{~F} / \mathrm{R}$.

\section{Establishment of a chemoresistant cell line}

The generated procedures of chemoresistant subline of $5-8 \mathrm{~F}$ were described and consulted in previous documents [26, 27]. The cisplatin (DDP)-resistant 5-8F subline was established by gradient induction. Briefly, 5-8F cells at log-growth phase were treated with $0.1 \mu \mathrm{g} / \mathrm{ml}$ DDP. Twenty-four hours later, DDP was removed and cells were washed by PBS. When cells' status returned to normal growth, they were passed for three generations followed by gradually increasing the concentration of DDP to 0.2, $0.5,1.0$ and $2.0 \mu \mathrm{g} / \mathrm{ml}$ until cells can maintain normal growth status. Those cells that can normally grow under the treatment of $2.0 \mu \mathrm{g} / \mathrm{ml}$ DDP were named as 5-8F/DDP.

\section{Cell viability assay prior to RNA interference (RNAi)}

Each group (5-8F/DDP, 5-8F/ $\mathrm{R}$ and the control 5-8/F) cells were seeded in 96-well plates at a density of $2 \times 10^{3}$ per well, and cultured at $37^{\circ} \mathrm{C}$ in $5 \% \mathrm{CO}_{2}$ for the indicated time $(24,48,72$ and $96 \mathrm{~h})$. At the different time points, cells were treated with DDP at different concentration $(0.5,1,2$ and $4 \mu \mathrm{g} / \mathrm{ml})$ or X-irradiation at different dose $(2,4,6$ and $8 \mathrm{~Gy})$, respectively. After treatment, the cells were incubated with $20 \mu 15 \mathrm{mg} / \mathrm{ml} \mathrm{3-(4,5-dimethylthiazol-2-yl)-2,5-}$ diphenyltetrazolium bromide (MTT) solution reagent (Sigma-Aldrich Corp.) for $4 \mathrm{~h}$, and then the cell viability was evaluated by MTT assay. The absorbance at $490 \mathrm{~nm}$ (A490) was measured using a microplate reader (Bio-Tek Inc., Winooski, VT, USA).

\section{Reverse transcription-quantitative PCR (RT-qPCR)}

Total RNA was extracted from cells using GeneJET $^{\text {TM }}$ RNA purification kit (Fermentas, Hanover, MD, USA) according to the manufacturer's instructions. With $1 \mu \mathrm{g}$ of total RNA used as a template, cDNA was synthesized using RevertAid ${ }^{\mathrm{TM}}$ first strand cDNA synthesis kit (Fermentas). PCR was performed using the following conditions: pre-denaturation at $94^{\circ} \mathrm{C}$ for $2 \mathrm{~min}$, followed by 35 cycles at $94^{\circ} \mathrm{C}$ for $30 \mathrm{~s}, 60^{\circ} \mathrm{C}$ for $30 \mathrm{~s}$ and $72^{\circ} \mathrm{C}$ for $30 \mathrm{~s}$, with a final extension at $72^{\circ} \mathrm{C}$ for $5 \mathrm{~min}$. Primer sequences used for RT-qPCR are listed in Table 1. The qPCR assays were performed using SYBR ${ }^{\mathrm{TM}}$ green PCR master mix (Applied Biosystems Inc., Foster City, CA, USA) on an ABI 7500 real-time PCR system (Applied Biosystems Inc.). GAPDH was used as the internal control. The relative mRNA expression levels were calculated using the $2^{-\Delta \Delta C t}$ method.

Table 1. Primer sequences used for RT-qPCR

\begin{tabular}{lll}
\hline Gene & Forward sequence (5'-3') & Reverse sequence (5'-3') \\
\hline SATB1 & GTGGAAGCCTTGGGAATCC & CTGACAGCTCTTCTTCTAGTT \\
MMP-9 & GCATCCGAGCAAGAAGACAA & CCCGACACACAGTAAGCATTC \\
& C & \\
E-cadheri & GAAGTGTCCGAGGACTTTGG & CAGTGTCTCTCCAAATCCGAT \\
$\mathrm{n}$ & & A \\
Vimentin & AGATGGCCCTTGACATTGAG & TGGAAGAGGCAGAGAAATTC \\
GAPDH & TCGGAGTCAACGGATTTGGT & TTGGAGGGATCTCGCTCCT \\
\hline
\end{tabular}

\section{Western blot (WB)}

Total protein was extracted using RIPA buffer (Sigma-Aldrich Corp.), and the protein concentrations were quantified by BCA protein assay kit (Boster Ltd., Wuhan, China). Total protein $(50 \mu \mathrm{g})$ was separated by $10 \%$ SDS-PAGE and transferred onto polyvinylidene fluoride membranes (PVDF; Millipore, Billerica, MA, USA). The membranes were incubated with polyclonal rabbit anti-SATB1 antibody (1:2,000; Proteintech, Chicago, IL, USA), and then incubated with horseradish peroxidase-conjugated secondary antibody (1:5,000; Proteintech). The ECL-based detection was performed with enhanced chemiluminescent reagent kit (Pierce Biotechnology Inc., Rockford, IL, USA) according to the manufacturer's instructions. Protein bands were visualized using the ECL plus detection system (Pierce Biotechnology Inc.). The level of a-tubulin was used as a loading control.

\section{Transfection}

SATB1 shRNA was designed and cloned into the pGFP-V-RS retroviral vector (Origene, Rockville, MD, USA). The SATB1-shRNA sequence was as follows: 5'-AGATTCAGCAGGAAATGAAGCGTGCTAAA-3'. The negative control shRNA sequence was as follows: 5'-AAGTCTTCTGACGCTGCTGCCTGGTCCAG-3' . Before transfection, $5-8 \mathrm{~F} / \mathrm{DDP}$ or $5-8 \mathrm{~F} / \mathrm{R}$ cells $(5 \times$ $10^{5}$ /well) were seeded in 6-well plates and grown in culture medium without antibiotics. For transfection, $4 \mu \mathrm{g}$ of the target plasmids and $10 \mu \mathrm{l}$ of Lipofectamine $^{\mathrm{TM}} 2000$ (Invitrogen) were diluted separately with $250 \mu \mathrm{l}$ of serum-free Opti-MEM ${ }^{\mathrm{TM}}$ (Gibco, Mulgrave, Victoria, Australia) medium, and then gently mixed. After $20 \mathrm{~min}$, the mixture was added into the seeded cells at $80 \%$ confluence. After 5 $\mathrm{h}$, the medium was replaced by normal medium. After $36 \mathrm{~h}$ incubation, the transfected cells were selected for 14 days with $0.5 \mu \mathrm{g} / \mathrm{ml}$ puromycin (Invitrogen). The cells with silenced SATB1 were designated as SATB1-shRNA group (SATB1-shRNA). Other groups were named as negative control group (NC-shRNA) and wild-type cell group (WT). 


\section{Cell proliferation assay after RNAi}

The cell proliferation ability was assessed by MTT assay. Each group (SATB1-shRNA, NC-shRNA and WT) cells were seeded in 96-well plates at a density of $2 \times 10^{3}$ per well, and cultured at $37^{\circ} \mathrm{C}$ in $5 \%$ $\mathrm{CO}_{2}$ for the indicated time $(24,48,72$ and $96 \mathrm{~h})$. At the different time points, cells were treated with $20 \mu \mathrm{l}$ MTT $(5 \mathrm{mg} / \mathrm{ml}$ ) solution reagent (Sigma-Aldrich Corp.). After incubation for $4 \mathrm{~h}$, A490 was measured using a microplate reader (Bio-Tek Inc.).

\section{Cell invasion assay after RNAi}

The cell invasion ability was determined using Matrigel Invasion Chamber (BD Biosciences, San Jose, CA, USA). In brief, cells in each group (SATB1shRNA, NC-shRNA and WT) were inoculated in the upper chamber at a density of $5 \times 10^{4}$ cells per $500 \mu \mathrm{l}$ per chamber and maintained in serum-free medium, and lower chamber were filled with $750 \mu$ l complete medium. Cells were incubated for $24 \mathrm{~h}$ at $37^{\circ} \mathrm{C}$ in a $5 \%$ $\mathrm{CO}_{2}$ incubator. The invaded cells were fixed with $4 \%$ paraformaldehyde, and then stained with $0.1 \%$ crystal violet. Five random independent fields were counted under light microscope.

\section{Drug resistance assay after $R N A i$}

After 5-8F/DDP cell was successfully transfected, each group (SATB1-shRNA, NC-shRNA and WT) cells were dispensed within 96-well plates at a density of $5 \times 10^{3}$ per well and cultured at $37^{\circ} \mathrm{C}$ in $5 \% \mathrm{CO}_{2}$. After $16 \mathrm{~h}$, the cells were treated with $10 \mu \mathrm{l} 1$ $\mu \mathrm{g} / \mathrm{ml}$ DDP. After $48 \mathrm{~h}$ incubation, cells were treated with $20 \mu \mathrm{l}$ MTT $(5 \mathrm{mg} / \mathrm{ml})$ solution reagent (Sigma-Aldrich Corp.) for $4 \mathrm{~h}$. The cell viability was assessed by MTT assay. A490 was measured using a microplate reader (Bio-Tek Inc.). The cell growth inhibition rate indirectly indicates the drug resistance ability of 5-8F/DDP cell to DDP.

\section{Radiation resistance assay after RNAi}

After $5-8 \mathrm{~F} / \mathrm{R}$ cell was successfully transfected, each group (SATB1-shRNA, NC-shRNA and WT) cells were dispensed within 96-well plates at a density of $5 \times 10^{3}$ per well and cultured at $37^{\circ} \mathrm{C}$ in $5 \% \mathrm{CO}_{2}$ for $16 \mathrm{~h}$. The cells were exposed to $4 \mathrm{~Gy}$ of X-ray for $48 \mathrm{~h}$ and then cells were treated with $20 \mu \mathrm{MTT}(5 \mathrm{mg} / \mathrm{ml})$ solution reagent (Sigma-Aldrich Corp.) for $4 \mathrm{~h}$. Cell viability was measured by MTT assay. A490 was assayed using a microplate reader (Bio-Tek Inc.). The cell growth inhibition rate indirectly indicates the radiation resistance ability of $5-8 \mathrm{~F} / \mathrm{R}$ cell to X-irradiation.

\section{Statistical analysis}

All measurement experiments were repeated three times independently, and the data are presented as mean \pm standard deviation (SD). Comparisons between two groups were performed using Student's $t$-test. Comparisons among multiple groups were performed using ANOVA. Statistical significance was defined as a $P$-value $<0.05$.

\section{Results}

\section{Chemoradiation resistance of 5-8F/DDP and 5-8F/R prior to SATB I knockdown}

MTT cell viability assay was used to determine the chemoradiation resistance of 5-8F/DDP and $5-8 \mathrm{~F} / \mathrm{R}$. The inhibitory effects of DDP on $5-8 \mathrm{~F}$ and $5-8 \mathrm{~F} / \mathrm{DDP}$ cells were investigated by varying incubation times at drug concentrations of $0.5,1,2$ and $4 \mu \mathrm{g} / \mathrm{ml}$. The inhibitory effects of X-ray on $5-8 \mathrm{~F}$ and $5-8 \mathrm{~F} / \mathrm{R}$ cells were investigated by varying incubation times at radiation doses of 2, 4, 6 and $8 \mathrm{~Gy}$. As illustrated in Figure 1A and 1B, the 5-8F cells showed gradually reduced cell viability when treated with serial increased dose of DDP and X-ray or exposed to the indicated dose at serial increased times. Thus, DDP and X-irradiation caused an attenuation in the cell viability of $5-8 \mathrm{~F}$ in a dose- and time-dependent manner, respectively. However, alteration of cell viability of $5-8 \mathrm{~F} / \mathrm{DDP}$ or $5-8 \mathrm{~F} / \mathrm{R}$ exposed to DDP or X-ray was not significant, respectively $(P>0.05)$ (Figure 1C, 1D).

\section{Altered expression of SATB 1, MMP-9, Vimentin and E-cadherin in chemoradiation resistant NPC cells}

RT-qPCR and WB analysis were used to assess the expression levels of SATB1, MMP-9, Vimentin and E-cadherin in 5-8F, 5-8F/DDP and 5-8F/R cells. RT-qPCR results showed that the mRNA expression levels of SATB1, MMP-9 and Vimentin in 5-8F/DDP and $5-8 \mathrm{~F} / \mathrm{R}$ were obviously higher than that in $5-8 \mathrm{~F}$ $(P<0.05)$ (Figure 2). Meanwhile, WB results showed that the protein expression levels of SATB1, MMP-9 and Vimentin in 5-8F/DDP and $5-8 \mathrm{~F} / \mathrm{R}$ were significantly increased than that in $5-8 \mathrm{~F} \quad(P<0.05)$ (Figure 3A, 3B). Nevertheless, the levels of mRNA and protein expression of E-cadherin in 5-8F/DDP and $5-8 \mathrm{~F} / \mathrm{R}$ were opposite compared with that in $5-8 \mathrm{~F}$ (Figure 2, 3A, 3B).

\section{SATB I was downregulated by RNAi in chemoradiation resistant NPC cells}

RT-qPCR and WB analysis were used to examine the silenced efficiency of SATB1 mRNA and protein expression in 5-8F/DDP and 5-8F/ $\mathrm{R}$ cell lines. The relative expression levels of SATB1 mRNA and protein in SATB1-shRNA cells were evidently downregulated than those in WT and NC-shRNA cells $(P<0.05)$ (Figure 4A, 4B, 5A-D), while the 
difference of relative expression levels of SATB1 mRNA and protein between WT group and NC-shRNA group was not significant $(P>0.05)$ (Figure 4A, 4B, 5A-D).

\section{SATB 1 knockdown inhibited proliferation of chemoradiation resistant NPC cells}

The proliferative ability of SATB1-shRNA, NC-shRNA and WT groups was examined by MTT method. The cell growth curve was drawn according to the A490 values at different time point, as shown in Figure 6A and 6B. The proliferation rate of SATB1-shRNA group was lower than NC-shRNA and WT groups from $48 \mathrm{~h}$, and the difference was significant $(P<0.05)$.

\section{SATB I knockdown inhibited invasion of chemoradiation resistant NPC cells}

In transwell assay, the transmembrane numbers of WT, SATB1-shRNA and NC-shRNA groups were shown in Table 2. Compared with NC-shRNA and WT groups, the transmembrane number of
SATB1-shRNA group was obviously decreased $(P<0.05)$ (Figure 7A, 7B).

Table 2. Quantification of invasion assay

\begin{tabular}{llll}
\hline Cell line & WT & SATB1-shRNA & NC-shRNA \\
\hline $5-8 \mathrm{~F} / \mathrm{DDP}$ & $58.33 \pm 4.51$ & $34.39 \pm 2.54$ & $57.34 \pm 3.07$ \\
$5-8 \mathrm{~F} / \mathrm{R}$ & $58.38 \pm 4.55$ & $34.66 \pm 2.61$ & $57.65 \pm 3.12$ \\
\hline
\end{tabular}

\section{SATB 1 knockdown modulated the expression of MMP-9 and EMT markers}

RT-qPCR and WB analysis were used to assess the expression levels of SATB1, MMP-9, Vimentin and E-cadherin in 5-8F/DDP and 5-8F/R cell lines. The mRNA and protein expression levels of MMP-9 and Vimentin were downregulated along with knockdown of SATB1 $(P<0.05)$ (Figure 4A, 4B, 5C, 5D). However, the mRNA and protein expression levels of E-cadherin were upregulated accompanied with knockdown of SATB1 $(P<0.05)$ (Figure 4A, 4B, $5 \mathrm{C}, 5 \mathrm{D})$.
A
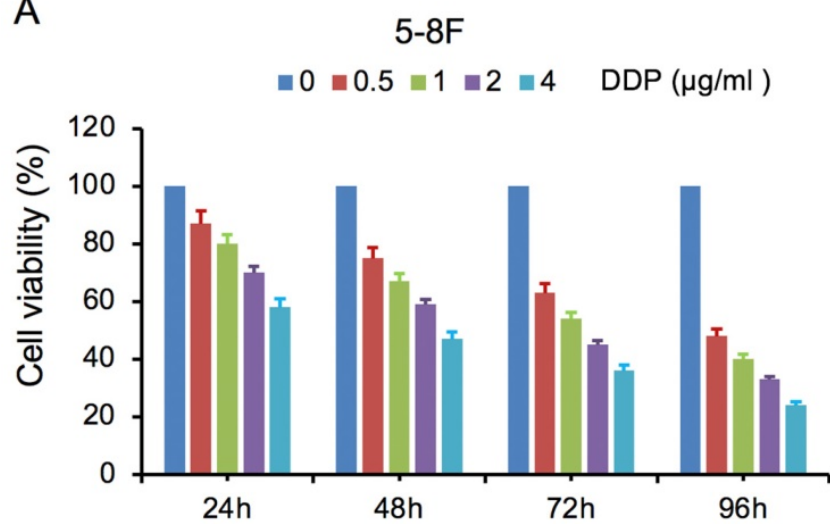

C

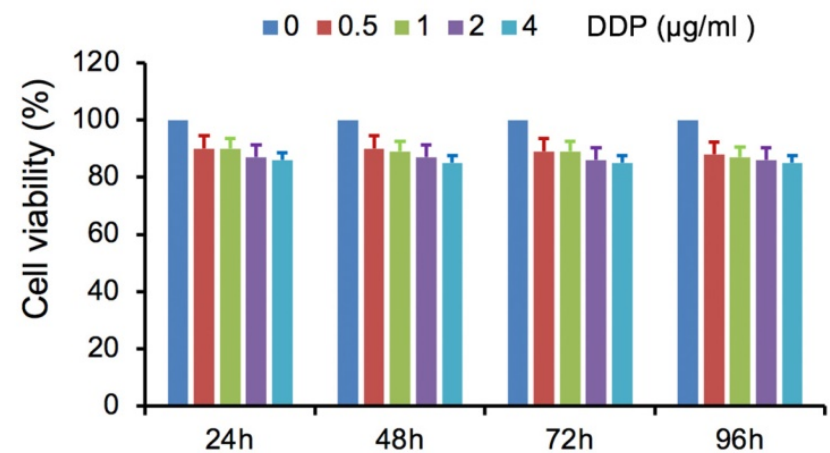

B

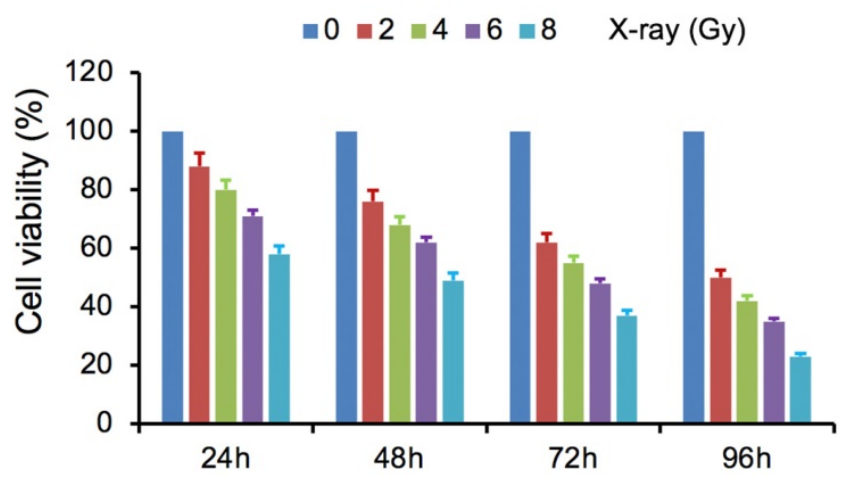

D

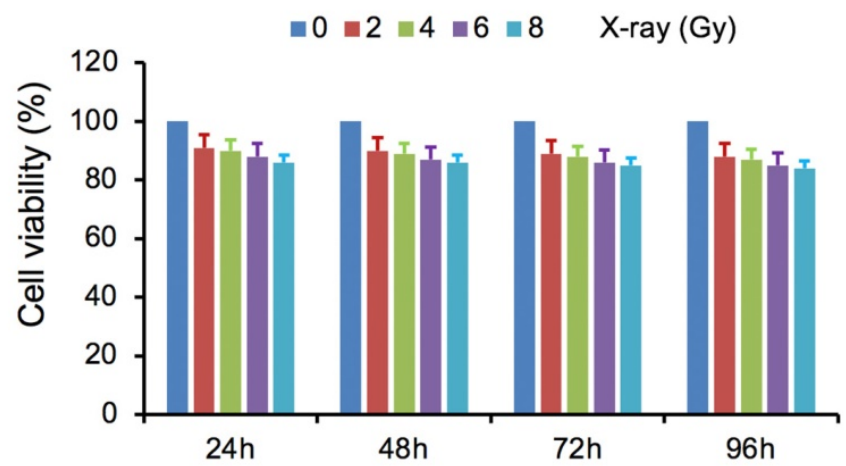

Figure 1. MTT cell viability assay showed that 5-8F/R and 5-8F/DDP were radioresistant or chemoresistant cells when compared to 5-8F cells. DDP (A) and X-irradiation (B) suppressed the cell viability of 5-8F in a dose- and time-dependent manner, respectively. 5-8F/DDP (C) or 5-8F/R (D) cells showed resistance to DDP or X-ray, respectively. 


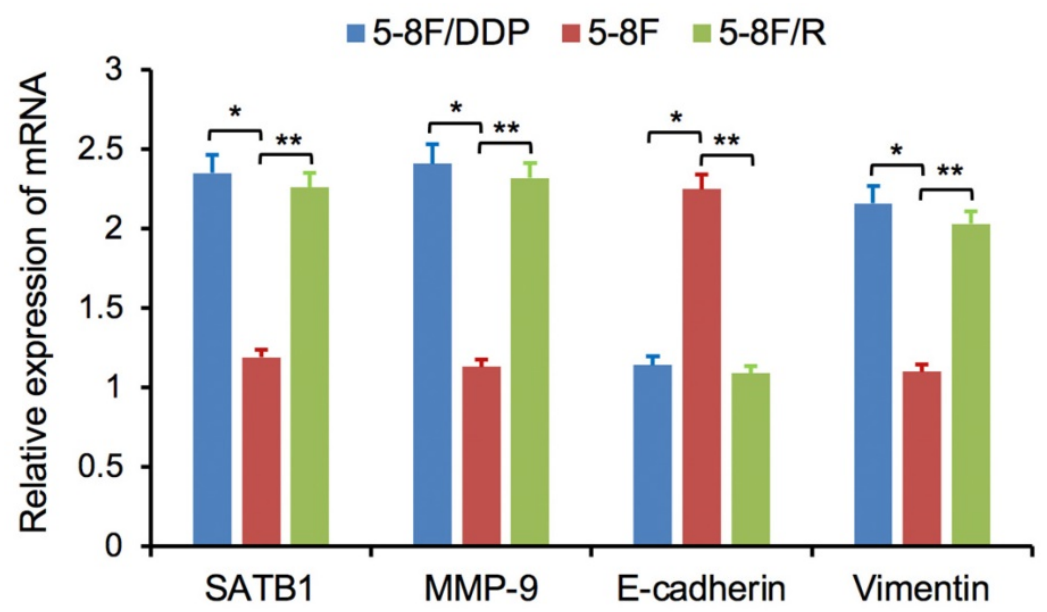

Figure 2. Expression of SATB1, MMP-9, Vimentin and E-cadherin genes in NPC cell lines by RT-qPCR. (* and **P<0.05 vs. 5-8F cell line).

A

\section{$5-8 \mathrm{~F} / \mathrm{DDP} \quad 5-8 \mathrm{~F} / \mathrm{R} \quad 5-8 \mathrm{~F}$}
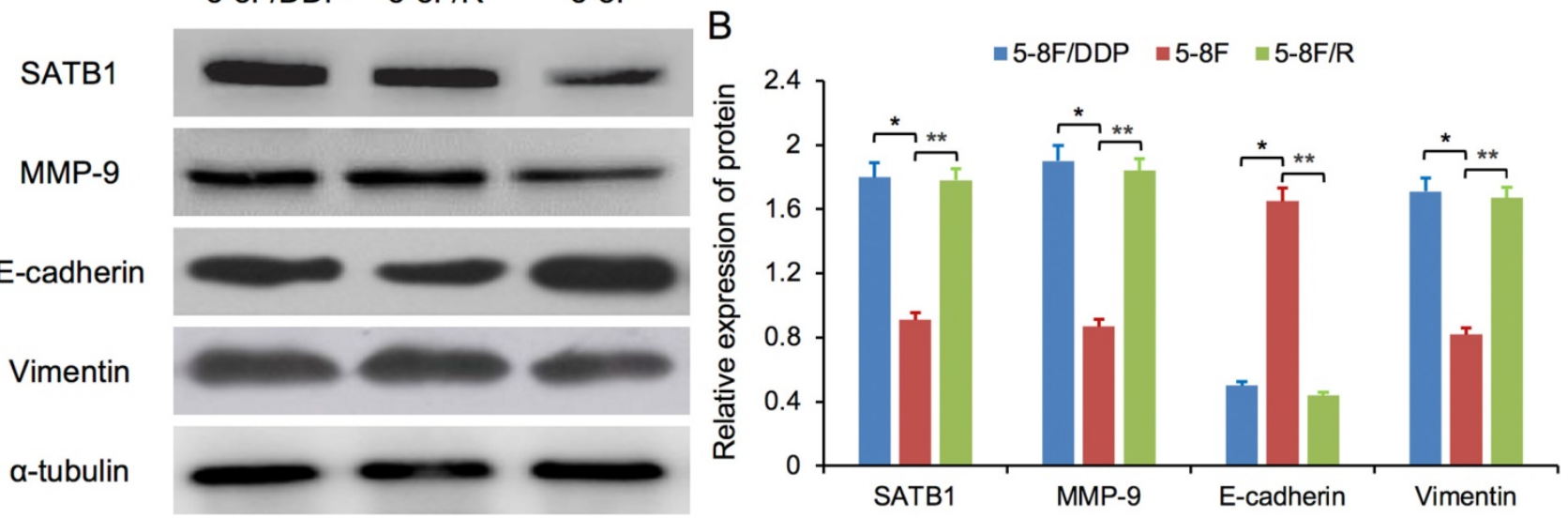

Figure 3. Expression of SATB1, MMP-9, Vimentin and E-cadherin in NPC cell lines by western blot analysis. (A) Representative protein electropherogram. (B) The relative protein expression levels of four genes normalized to that of $\alpha$-tubulin. (* and $* * P<0.05$ vs. $5-8 \mathrm{~F}$ cell line).
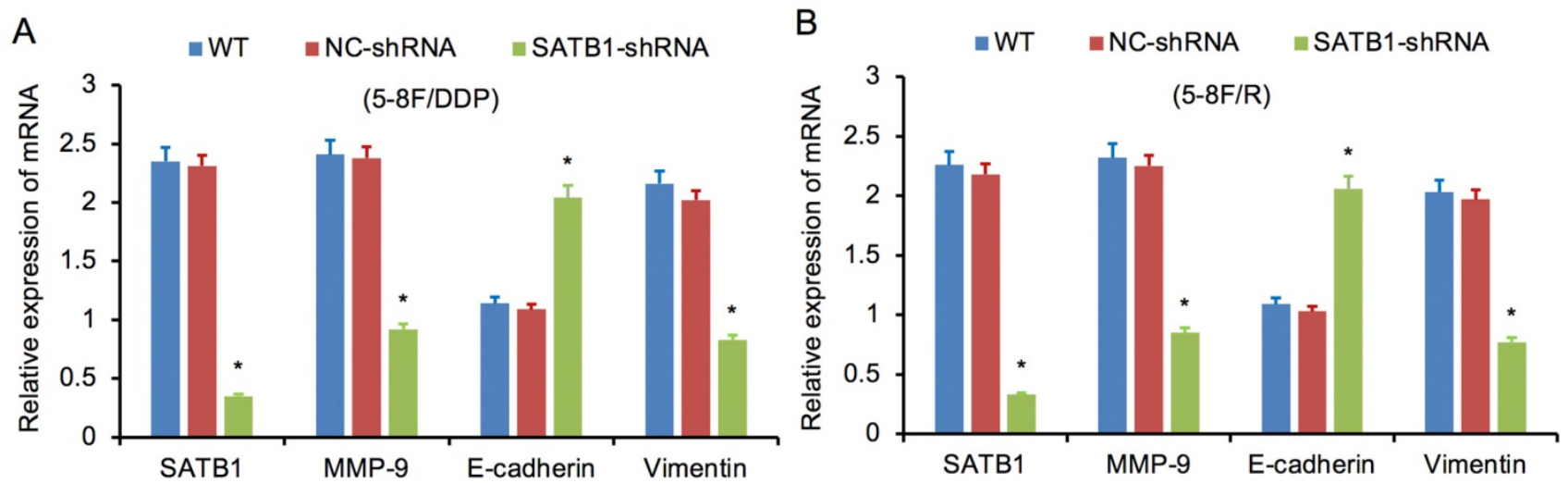

Figure 4. SATB1 knockdown regulated the expression of MMP-9, Vimentin and E-cadherin. (A) Relative mRNA expression levels of four genes in 5-8F/DDP cells. (B) Relative mRNA expression levels of four genes in 5-8F/R cells. WT, wild-type cell group (the untreated 5-8F/DDP or 5-8F/R cells). NC-shRNA, negative control group (the cells were transfected with negative control shRNA). ( ${ }^{* P}<0.05$ vs. WT and NC-shRNA). 
A

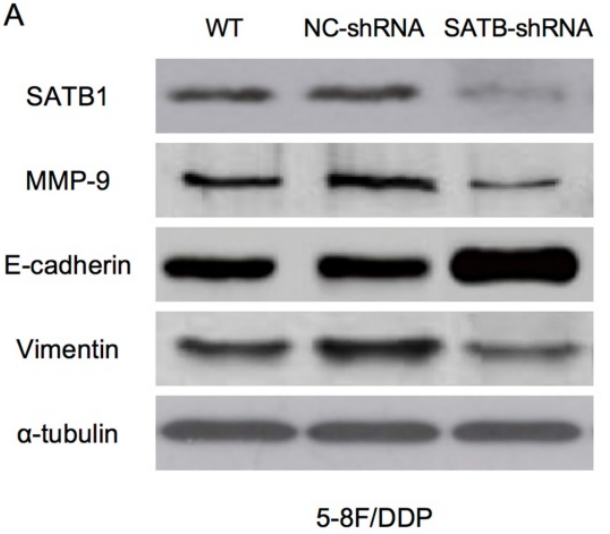

C
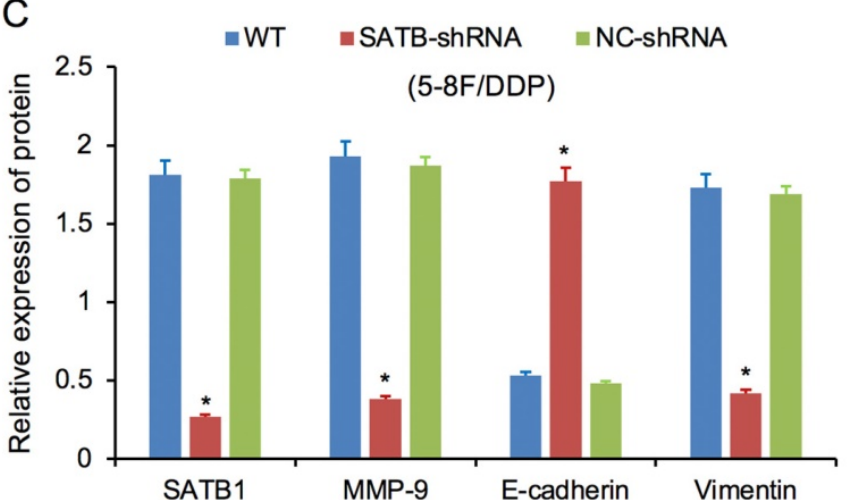

B

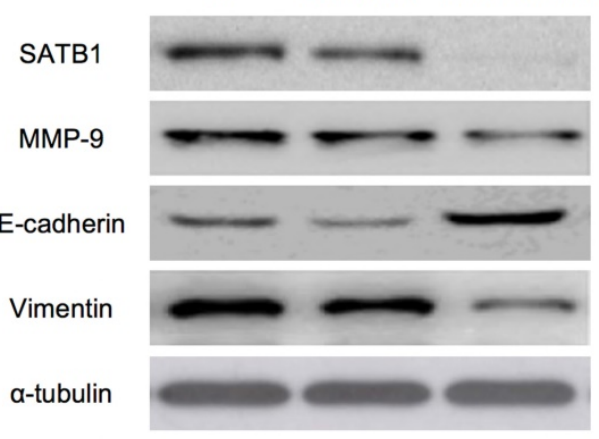

D

Figure 5. Expression of SATB1, MMP-9, Vimentin and E-cadherin in different groups by western blot analysis. (A) Representative protein electropherogram of 5-8F/DDP cells. (B) Representative protein electropherogram of 5-8F/R cells. (C) The relative protein expression levels of four genes normalized to that of $\alpha$-tubulin in 5-8F/DDP cells. (D) The relative protein expression levels of four genes normalized to that of $\alpha$-tubulin in $5-8 F / R$ cells. $(* P<0.05$ vs. WT and NC-shRNA).
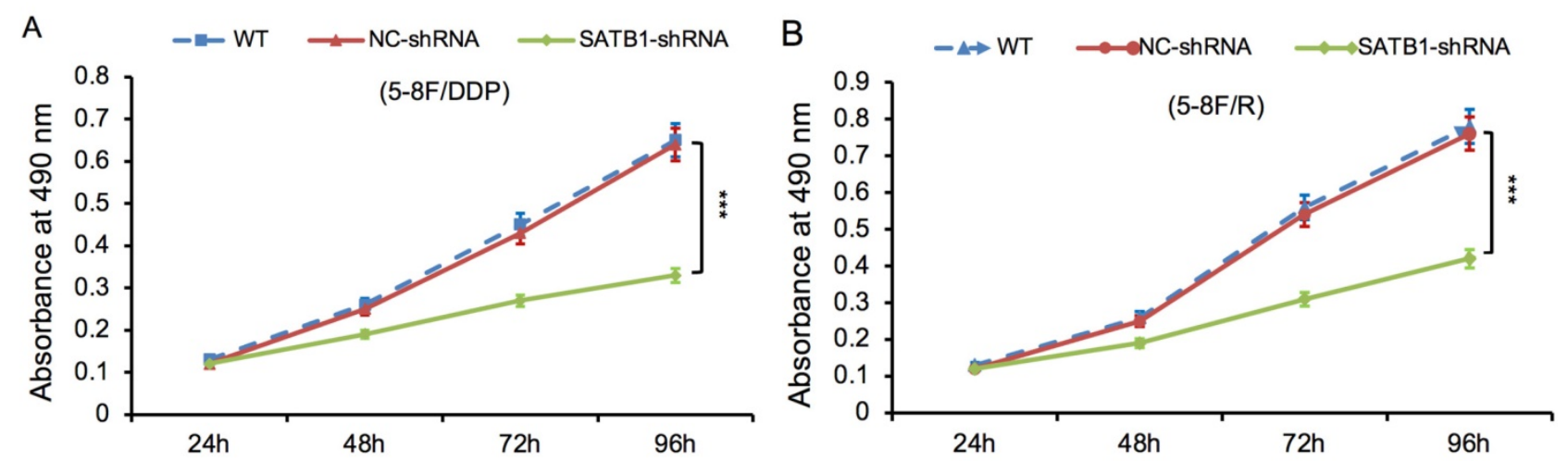

Figure 6. SATB1 knockdown inhibited proliferation in chemoradiation resistant NPC cell lines at different time-points (A) 5-8F/DDP cells and (B) 5-8F/R cells. Graph shows cell growth curve was drawn according to the $A 490$ values at different time-points. (*** $P<0.05$ vs. WT and NC-shRNA).
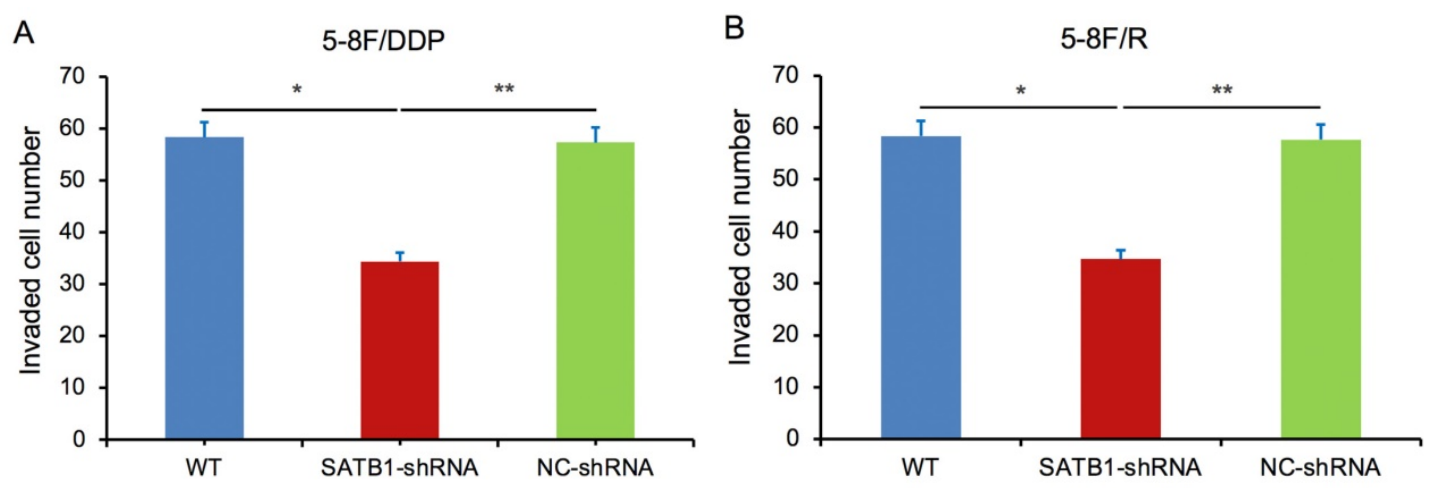

Figure 7. SATB1 knockdown inhibited invasion of chemoradiation resistant NPC cell lines (A) 5-8F/DDP cells and (B) 5-8F/R cells. Graph shows the number of invaded cells in the treatment groups for respective cell lines. (* and $* * P<0.05$ vs. SATB1-shRNA). 


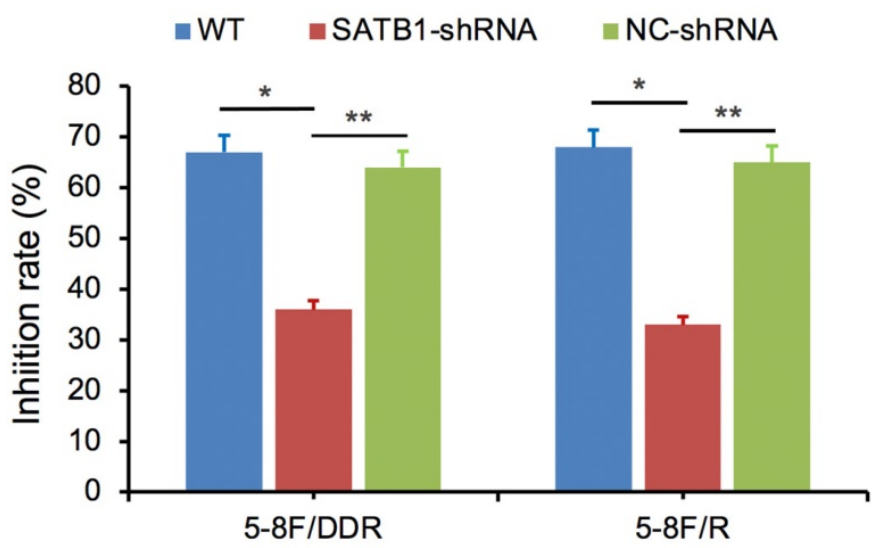

Figure 8. SATB1 knockdown decreased 5-8F/DDP cells resistance to DDP and retarded 5-8F/R cells resistance to X-irradiation. (* and **P<0.05 vs. SATB1-shRNA).

\section{SATB 1 knockdown decreased 5-8F/DDP cell resistance to DDP and retarded 5-8F/R cell resistance to $\mathrm{X}$-irradiation}

SATB1-silencing 5-8F/DDP or 5-8F/R cells (SATB1-shRNA), negative control cells (NC-shRNA) and WT cells were exposed individually to DDP (1 $\mu \mathrm{g} / \mathrm{ml}$ ) or X-irradiation (4 Gy). Cell viability was examined after $48 \mathrm{~h}$ and then the growth inhibition rate was calculated. Compared with NC-shRNA and WT groups, the inhibition rate of SATB1-shRNA group was significantly decreased $(P<0.05)$ (Figure 8).

\section{Discussion}

NPC is one of the common malignancies of epithelial origin in head and neck cancers [2, 28]. Chemoradiotherapy is main treatment option for many epithelial tumor types including NPC, but the effectiveness of chemoradiotherapy is limited owing to radiation resistance and drug resistance $[4,6]$. Currently, there are few effective biomarkers available in the clinic for predicting tumor chemoradiosensitivity $[1,29,30]$. To elucidate the underlying mechanism and find the novel therapeutic targets, the radioresistant and drug resistant cell lines were urgently needed. A DDP-resistant cell line $5-8 \mathrm{~F} / \mathrm{DDP}$ and a radioresistant cell line $5-8 \mathrm{~F} / \mathrm{R}$ have been established from human NPC cell line 5-8F. It is convenient for us to carry out subsequent experiments.

SATB1, a tissue-specific nuclear matrix-attachment DNA binding protein, is involved in chromatin structure packaging and gene expression and regulates numerous cellular processes such as differentiation, proliferation and apoptosis through effects on downstream gene expression [18, 31]. SATB1 is an identified oncogene and its increased expression is associated with tumor growth, metastasis and poor prognosis in various cancers $[7,8$, 32]. Furthermore, it is an independent prognostic marker across several cancers [10, 33]. Our previous study found that the expression levels of SATB1 were significantly upregulated in primary NPC tissues and NPC cell line 5-8F, and aberrant SATB1 expression was connected with Epstein-Barr virus (EBV) infection, metastasis and survival in NPC patients [13]. Another investigation by our group indicated that SATB1 may be involved in the development, progression and drug resistance of NPC cell line CNE-2 [14]. Additionally, few works have demonstrated that STAB1 was closely associated with drug resistant and multidrug resistance in gastric cancer, breast cancer and human glioblastoma [11, 12, 34]. To date, as we knowledge, no data on SATB1 and radioresistance of NPC were reported. In the present study, our results have displayed SATB1 expression levels of mRNA and protein were significantly elevated in NPC chemoradiation resistant cells $5-8 \mathrm{~F} / \mathrm{R}$ and $5-8 \mathrm{~F} / \mathrm{DDP}$ compared with 5-8F. This suggests that upregulated SATB1 may be induce the drug resistance and radiation resistance of NPC cells.

In next experiments, we successfully silenced the SATB1 expression in chemoradioresistant cell line $5-8$ F/DDP and 5-8F/R by shRNA. RT-qPCR and WB analysis showed the expression of SATB1 were distinctly downregulated after RNAi. MTT and transwell assays exhibited the capabilities of cell proliferation and invasion were visibly decreased after SABT1 knockdown. These results implicate that SATB1 promotes the growth and metastasis of NPC cells and successful silencing of SATB1 can prevent malignant behavior of NPC cells.

MMP-9 has been widely found to relate to the pathology of cancers, including but not limited to growth, invasion, metastasis and angiogenesis [21, 35, 36]. Besides, previous works found that downregulation of MMP-9 can improve the chemosensitivity and radiosensitivity of some cancers $[22,23,37]$. Next, recent studies indicated MMP-9 is downstream target molecule of some genes or 
signaling pathway, such as NF-KB, HIF, LINC00511 $[23,38,39]$. In this study, we found that MMP-9 was highly expressed in chemoradiation resistant NPC cells $5-8 \mathrm{~F} / \mathrm{R}$ and 5-8F/DDP compared with 5-8F, whereas its expression was remarkably decreased concomitant with silencing of SATB1. Above findings demonstrated that successful knockdown of STAB1 can suppress the expression of MMP-9 and MMP-9 may be the downstream target effector of STAB1.

Furthermore, MMP-9 has been shown to be involved in the progression of NPC via facilitating tumor growth, invasion and metastasis [37, 40, 41]. Our previous study also found upregulated MMP-9 protein expression in NPC tissue was closely associated with EBV infection, metastasis, recurrence and poor survival of NPC patients [42]. The present results first confirmed that abnormal MMP-9 expression contributes to cancer cell proliferation and invasion of chemoradiation resistant NPC.

In addition, various molecules including MMP-9 are known to regulate the EMT [24]. Combined with our results regarding to MMP-9 and chemoradiation resistant NPC, it has been implicated that MMP-9 may be linked with NPC chemoresistance via participating in EMT processes [37, 43]. Accumulating data have shown that EMT is closely associated with invasion, metastasis, radioresistance and chemoresistance in many cancers, including NPC [5, 17, 44]. Previous study has documented that the development of DDP resistance in human NPC cells is accompanied by inducible EMT-like changes with an increased metastatic potential in vitro [43].

The reversible process of EMT is named as MET, which would allow transformed mesenchymal-like cells to regain their epithelial characteristics, a state that is perhaps more advantageous for survival in a foreign microenvironment [45]. Reversion of expression of epithelial-related genes (e.g. E-cadherin) and mesenchymal-related genes (e.g. Vimentin) is the principal characteristic of EMT/MET transition. It has been reported that depletion of TAZ (Transcriptional co-activator with PDZ binding motif) reversed the EMT features to MET and restored the DDP sensitivity in resistant NPC cells [46]. In the current study, high expression of Vimentin and low expression of E-cadherin in chemoradiation resistant NPC cells 5-8F/DDP and 5-8F/R were dramatically reversed along with knockdown of SATB1, and it was consistent with the alteration of MMP-9 expression. Thus, our results revealed that altered and reversed expression of MMP-9 and important EMT markers (E-cadherin and Vimentin) in chemoradiation resistant NPC cells, validating the roles of MMP-9 and EMT in SATB1-induced chemoradiation resistance of NPC. Taken together, these findings implied that depletion of SATB1 may reversed the EMT to MET situation by regulating its downstream genes controlling EMT processes.

Many studies have hinted the relationships among SATB1, MMP-9 and EMT. Han and his groups reported that SATB1 upregulated the expression of MMP-9 in breast cancer [18]. A recent study found that SATB1 plays a crucial role in the progression of bladder cancer by regulating genes controlling EMT processes [20]. SATB1 has also been identified to promote drug-induced EMT in breast cancer cell lines, driven by the positive feedback regulation of miR-448 and NF-kB signaling [47]. Another study suggested that SATB1 may play an important role in the development and progression of liver cancer by main regulation of EMT [48]. It's reported that SATB1 was able to promote the metastasis of prostate cancer by modulation of EMT [49]. Moreover, Frömberg and colleagues revealed that knockdown of SATB1 in colorectal cell lines can simultaneously influence the expression of manifold genes, which involve EMT and matrix breakdown [50].

Therefore, we hypothesized that there may be a crucial regulatory relationship between SATB1 and MMP-9 and EMT during the formation of tumor growth, metastasis and chemoradioresistance in NPC. Our experiments have identified that SATB1, MMP-9 and Vimentin were upregulated in chemoradiation resistant NPC cells 5-8F/DDP and 5-8F/ R compared with $5-8 \mathrm{~F}$, whereas E-cadherin was downregulated. Next works have confirmed that SATB1 knockdown in DDP-resistant and X-radiation resistant NPC cells caused downregulated expression of MMP-9 and Vimentin and increased expression of E-cadherin, and repressed cell proliferative and invasive ability. So, we deem SATB1 could induce chemoradioresistance of NPC cells and facilitate growth and metastasis of NPC through modulating MMP-9 expression and EMT process.

Additionally, the current results exhibited that DDP and X-ray individually did not prominently inhibit cell viability of 5-8F/DDP and 5-8F/ $R$ without SATB1 knockdown regardless of the increase of treatment dose and time. $5-8 \mathrm{~F} / \mathrm{DDP}$ or $5-8 \mathrm{~F} / \mathrm{R}$ cells showed resistance to DDP or X-ray, respectively. This is different from the effects of DDP and X-ray on non-chemoradiation resistant tumor cells $5-8 \mathrm{~F}$, which in a dose- and time-dependent manner. These data indicated drug or X-ray was difficult to improve therapeutic effect by increasing the dose of drug and $X$-irradiation when appearance of chemoradioresistance in NPC cells. It is helpful for clinical treatment decisions.

Subsequently, drug and radiation resistance assays demonstrated that cell viability of 5-8F/DDP 
and 5-8F/R with SATB1 knockdown was suppressed when 5-8F/DDP cell were treated with indicated DDP concentrations as well as the same situation in 5-8F/ R cell treated with indicated $\mathrm{X}$-ray dose. These results present that silencing of SATB1 can diminished drug resistance and radiation resistance in NPC cells.

In summary, SATB1 has been shown to contribute to tumor growth, metastasis, drug resistance and radiation resistance in NPC, and the possible mechanism is developed that SATB1 could simultaneously modulate multiple downstream factors, thus regulating tumor cell proliferation, invasion, chemoradiation resistance and EMT/MET switch. To the best of our knowledge, this is the first time that we here clarify the correlation between SATB1 and radiation resistance in NPC. Overall, our data indicate that SATB1 may be a promising therapeutic target and useful biomarker for chemoradiation resistant and aggressive NPC patients. Further studies are required to investigate clinical application value of SATB1 as the therapeutic target and predictive marker for NPC.

\section{Acknowledgements}

This work was supported by the Natural Science Fund of Fujian Province, China (No. 2017J01374).

\section{Ethics Statement}

All experiments were conducted in accordance with the ethical guidelines and protocols were approved by the Medical Ethics Committee of Zhongshan Hospital, Xiamen University.

\section{Competing Interests}

The authors have declared that no competing interest exists.

\section{References}

1. Tsang CM, Lui VWY, Bruce JP, et al. Translational genomics of nasopharyngeal cancer. Semin Cancer Biol. 2020; 61: 84-100.

2. Chen YP, Chan ATC, Le QT, et al. Nasopharyngeal carcinoma. Lancet. 2019; 394: 64-80.

3. Lee $\mathrm{AW}, \mathrm{Ma} \mathrm{BB}, \mathrm{Ng} \mathrm{WT}$, et al. Management of nasopharyngeal carcinoma: current practice and future perspective. J Clin Oncol. 2015; 33: 3356-64.

4. Perri F, Della Vittoria Scarpati G, Caponigro F, et al. Management of recurrent nasopharyngeal carcinoma: current perspectives. Onco Targets Ther. 2019; 12: 1583-91.

5. Lu Y, Liang Y, Zheng X, et al. EVI1 promotes epithelial-to-mesenchymal transition, cancer stem cell features and chemo-/radioresistance in nasopharyngeal carcinoma. J Exp Clin Cancer Res. 2019; 38: 82.

6. Chen C, Chen T, Huang C, et al. Experience of weekly cisplatin concurrent with intensity-modulated radiotherapy for locally advanced nasopharyngeal carcinoma patients with resistance to neoadjuvant chemotherapy. Medicine. 2017; 96: e8434.

7. Fredholm S, Willerslev-Olsen A, Met $\mathrm{O}$, et al. SATB1 in malignant $\mathrm{T}$ cells. J Invest Dermatol. 2018; 138: 1805-15.

8. Fromberg A, Engeland K, Aigner A. The special AT-rich sequence binding protein 1 (SATB1) and its role in solid tumors. Cancer Lett. 2018; 417: 96-111.

9. Glatzel-Plucinska N, Piotrowska A, Dziegiel P, et al. The role of SATB1 in tumour progression and metastasis. Int J Mol Sci. 2019; 20: 4156

10. Hedner C, Gaber A, Korkocic D, et al. SATB1 is an independent prognostic factor in radically resected upper gastrointestinal tract adenocarcinoma. Virchows Arch. 2014; 465: 649-59.
11. $\mathrm{Li} \mathrm{QQ}, \mathrm{Chen} Z \mathrm{Z}, \mathrm{Xu} J \mathrm{D}$, et al. Overexpression and involvement of special AT-rich sequence binding protein 1 in multidrug resistance in human breast carcinoma cells. Cancer Sci. 2010; 101: 80-6.

12. Sun $\mathrm{F}, \mathrm{Lu} \mathrm{X}, \mathrm{Li} \mathrm{H}$, et al. Special AT-rich sequence binding protein 1 regulates the multidrug resistance and invasion of human gastric cancer cells. Oncol Lett. 2012; 4: 156-62.

13. Deng YF, Zhou DN, Pan ZY, et al. Aberrant SATB1 expression is associated with Epstein-Barr virus infection, metastasis and survival in human nasopharyngeal cells and endemic nasopharyngeal carcinoma. Int J Clin Exp Pathol. 2014; 7: 2454-61.

14. Ye CS, Zhou DN, Yang QQ, et al. Silencing SATB1 influences cell invasion, migration, proliferation, and drug resistance in nasopharyngeal carcinoma. Int J Clin Exp Pathol. 2014; 7: 914-22.

15. Du F, Liu H, Lu Y, et al. Epithelial-to-mesenchymal transition: liaison between cancer metastasis and drug resistance. Crit Rev Oncog. 2017; 22: 275-82.

16. Huang $\mathrm{T}$, Yin $\mathrm{L}, \mathrm{Wu}$, et al. TNFAIP3 inhibits migration and invasion in nasopharyngeal carcinoma by suppressing epithelial mesenchymal transition. Neoplasma. 2017; 64: 389-94.

17. Dongre A, Weinberg RA. New insights into the mechanisms of epithelial-mesenchymal transition and implications for cancer. Nat Rev Mol Cell Biol. 2019; 20: 69-84.

18. Han HJ, Russo J, Kohwi Y, et al. SATB1 reprogrammes gene expression to promote breast tumour growth and metastasis. Nature. 2008; 452: 187-93.

19. Qi H, Fu X, Li Y, et al. SATB1 promotes epithelial-mesenchymal transition and metastasis in prostate cancer. Oncol Lett. 2017; 13: 2577-82.

20. Wan F, Cheng C, Wang Z, et al. SATB1 overexpression regulates the development and progression in bladder cancer through EMT. PLoS One. 2015; 10: e0117518.

21. Huang H. Matrix metalloproteinase-9 (MMP-9) as a cancer biomarker and MMP-9 biosensors: recent advances. Sensors (Basel). 2018; 18: 3249.

22. Asuthkar S, Velpula KK, Chetty C, et al. Epigenetic regulation of miRNA-211 by MMP-9 governs glioma cell apoptosis, chemosensitivity and radiosensitivity. Oncotarget. 2012; 3: 1439-54.

23. Kunigal S, Lakka SS, Joseph P, et al. Matrix metalloproteinase-9 inhibition down-regulates radiation-induced nuclear factor-kappa B activity leading to apoptosis in breast tumors. Clin Cancer Res. 2008; 14: 3617-26.

24. Agraval H, Yadav UCS. MMP-2 and MMP-9 mediate cigarette smoke extract-induced epithelial-mesenchymal transition in airway epithelial cells via EGFR/Akt/GSK3beta/beta-catenin pathway: amelioration by fisetin. Chem Biol Interact. 2019; 314: 108846.

25. Asuthkar S, Nalla AK, Gondi CS, et al. Gadd45a sensitizes medulloblastoma cells to irradiation and suppresses MMP-9-mediated EMT. Neuro Oncol. 2011; 13: 1059-73.

26. Wu P, Tang Y, He J, et al. ARC is highly expressed in nasopharyngeal carcinoma and confers X-radiation and cisplatin resistance. Oncol Rep. 2013; 30: 1807-13.

27. Jing D, Zhou W, Shen L, et al. RIG-I promotes IFN/JAK2 expression and the endoplasmic reticulum stress response to inhibit chemoradiation resistance in nasopharyngeal carcinoma. Cancer Med. 2019; 8: 6344-57.

28. Paul P, Deka H, Malakar AK, et al. Nasopharyngeal carcinoma: understanding its molecular biology at a fine scale. Eur J Cancer Prev. 2018; 27: 33-41.

29. Kang H, Kiess A, Chung CH. Emerging biomarkers in head and neck cancer in the era of genomics. Nat Rev Clin Oncol. 2015; 12: 11-26.

30. Grade M, Wolff HA, Gaedcke J, et al. The molecular basis of chemoradiosensitivity in rectal cancer: implications for personalized therapies. Langenbecks Arch Surg. 2012; 397: 543-55.

31. Naik R, Galande S. SATB family chromatin organizers as master regulators of tumor progression. Oncogene. 2019; 38: 1989-2004.

32. Wang S, Zeng J, Xiao R, et al. Poor prognosis and SATB1 overexpression in solid tumors: a meta-analysis. Cancer Manag Res. 2018; 10: 1471-8.

33. Lu X, Cheng C, Zhu S, et al. SATB1 is an independent prognostic marker for gastric cancer in a Chinese population. Oncol Rep. 2010; 24: 981-7.

34. Yang B, Ma YB, Chu SH. Silencing SATB1 overcomes temozolomide resistance by downregulating MGMT expression and upregulating SLC22A18 expression in human glioblastoma cells. Cancer Gene Ther. 2018; 25: 309-16.

35. Klein G, Vellenga E, Fraaije MW, et al. The possible role of matrix metalloproteinase (MMP)-2 and MMP-9 in cancer, e.g. acute leukemia. Crit Rev Oncol Hematol. 2004; 50: 87-100.

36. Wang J, Shi Q, Yuan TX, et al. Matrix metalloproteinase 9 (MMP-9) in osteosarcoma: review and meta-analysis. Clin Chim Acta. 2014; 433: 225-31.

37. Sun XJ, Zhang P, Li HH, et al. Cisplatin combined with metformin inhibits migration and invasion of human nasopharyngeal carcinoma cells by regulating E-cadherin and MMP-9. Asian Pac J Cancer Prev. 2014; 15: 4019-23.

38. Mao BD, Xu P, Xu P, et al. LINC00511 knockdown prevents cervical cancer cell proliferation and reduces resistance to paclitaxel. J Biosci. 2019; 44: 44.

39. Meng L, Cheng Y, Tong X, et al. Tumor oxygenation and hypoxia inducible factor-1 functional inhibition via a reactive oxygen species responsive nanoplatform for enhancing radiation therapy and abscopal effects. ACS Nano. 2018; 12: 8308-22.

40. Liao D, Huang H, Zhu Z, et al. Prognostic value of matrix metalloproteinase 9 in nasopharyngeal carcinoma: a meta-analysis. Minerva Med. 2016; 107: 54-61.

41. Das AK, Baishya N, Sarma A, et al. Assessment and clinicopathological correlation of matrix metalloproteinase 9 expression in nasopharyngeal carcinoma. J Carcinog. 2019; 18: 1. 
42. Zhou DN, Deng YF, Li RH, et al. Concurrent alterations of RAGE, RECK, and MMP9 protein expression are relevant to Epstein-Barr virus infection, metastasis, and survival in nasopharyngeal carcinoma. Int J Clin Exp Pathol. 2014; 7: 3245-54.

43. Zhang $\mathrm{P}$, Liu H, Xia F, et al. Epithelial-mesenchymal transition is necessary for acquired resistance to cisplatin and increases the metastatic potential of nasopharyngeal carcinoma cells. Int J Mol Med. 2014; 33: 151-9.

44. Su Z, Li G, Liu C, et al. Autophagy inhibition impairs the epithelial-mesenchymal transition and enhances cisplatin sensitivity in nasopharyngeal carcinoma. Oncol Lett. 2017; 13: 4147-54.

45. Carstens JL, Lovisa S, Kalluri R. Microenvironment-dependent cues trigger miRNA-regulated feedback loop to facilitate the EMT/MET switch. J Clin Invest. 2014; 124: 1458-60.

46. Li S, Zhang X, Zhang $\mathrm{R}$, et al. Hippo pathway contributes to cisplatin resistant-induced EMT in nasopharyngeal carcinoma cells. Cell Cycle. 2017; 16: 1601-10.

47. $\mathrm{Li} \mathrm{OQ}$, Chen $\mathrm{ZQ}$, Cao XX, et al. Involvement of NF-kappaB/miR-448 regulatory feedback loop in chemotherapy-induced epithelial-mesenchymal transition of breast cancer cells. Cell Death Differ. 2011; 18: 16-25.

48. Tu W, Luo M, Wang Z, et al. Upregulation of SATB1 promotes tumor growth and metastasis in liver cancer. Liver Int. 2012; 32: 1064-78.

49. Mao LJ, Yang CH, Fan L, et al. SATB1 promotes prostate cancer metastasis by the regulation of epithelial-mesenchymal transition. Biomed Pharmacother. 2016; 79: 1-8.

50. Fromberg A, Rabe M, Aigner A. Multiple effects of the special AT-rich binding protein 1 (SATB1) in colon carcinoma. Int J Cancer. 2014; 135: 2537-46. 